\title{
Abundance and Diversity of Microbiota in Type 2 Diabetes and Obesity
}

Marlene Remely¹, Simone Dworzak¹, Berit Hippe ${ }^{1}$, Jutta Zwielehner ${ }^{1}$, Eva Aumüller ${ }^{1}$, Helmut Brath² and Alexander Haslberger ${ }^{1 *}$

${ }^{1}$ Department of Nutritional Sciences, Vienna, Austria

2Diabetes Outpatient Clinic, Health Center South, Vienna, Austria

\begin{abstract}
Inflammatory reactions leading to the progression of metabolic syndrome contribute to changes in composition of the GIT microbiota.

We characterize fecal microbiota at three time points in fourteen obese participants, nineteen lean controls and twenty-four type 2 diabetes patients. Obese and type 2 diabetics received an intervention of nutritional counseling, type 2 diabetics an additional therapy with a GLP-1-Agonist. The microbiota composition was analyzed for abundance and diversity by quantitative real-time polymerase chain reaction, denaturing gradient gel electrophoresis and high throughput sequencing.
\end{abstract}

In type 2 diabetics an increase of diversity was observed with intervention whereas the values of lean controls remained unaffected. In the lean and obesity groups, a lower Firmicutes:Bacteroidetes ratio correlated with lower BMI. In type 2 diabetics the ratio of Firmicutes to Bacteroidetes increased throughout the intervention period. Type 2 diabetics showed a significantly enhanced proportion of lactic acid bacteria before and after intervention, also Akkermansia and Enterobacteria showed a higher abundance in type 2 diabetics, increasing throughout the study period. Archaea were significantly more frequent in type 2 diabetics.

Whether differences seen in the abundance of certain groups and in the diversity of microbiota reflect different underlying inflammatory mechanisms of type 2 diabetes and obesity or rather the progression of the metabolic disease remains unclear and needs long-term investigation.

Keywords: GLP-1-Agonist; Metabolic syndrome; Ratio Firmicutes:Bacteroidetes; Lactic acid bacteria; Akkermansia; Prevotella; Enterotypes

Abbreviations: ANOVA: Analysis of Variance; AMPK: AMP-Activated Protein Kinase; BMI: Body Mass Index; DGGE: Denaturing Gradient Gel Electrophoresis; DSMZ: German collection of Microorganisms and Cell Cultures; FFG: The Austrian Research Promotion Agency; FFQ: Food Frequency Questionnaire; FIAF Fasting-Induced Adipose Factor (or Angiopoietin-like Protein 4); GI: Gastro Intestinal; GLP: Glucagon-Like Peptide; Gpr: G-Protein Coupled Receptor; $\mathrm{HbA}_{1}$ : Glycol Hemoglobin; IL: Interleukin; LAB: Lactic Acid Bacteria; LPS: Lipopolysaccharide; MCP-1: Monocyte Chemo Attractant Protein-1; P: Probability; PAI-1 - Plasminogen Activator Inhibitor-1; PC: Principal Component; PCA: Principal Component Analysis; PCR: Polymerase Chain Reaction; PYY: Peptide YY; qPCR: Quantitative Polymerase Chain Reaction; rDNA: Ribosomal DNA; SCFA: Short Chain Fatty Acid; T: Time Point; TLR: Toll Like Receptor; TNFa: Tumor Necrosis Factor Alpha; UPGMA: Unweighted Pair Group Method with Arithmetic Mean; UK: United Kingdom

\section{Introduction}

The gut microbiota an integral part of a complex network coordinating the physical and chemical elements of the intestinal barrier together with the immune, sensory, neuromotor- and enteroendocrine systems [1]. It bridges the indeterminate gap between food and weight by affecting the host metabolism through regulation of intestinal glucose absorption, lipogenesis and fat deposition. Housekeeping functions such as the central carbon metabolism or the amino acid synthesis of important protein complexes are present in every bacterium. Secondary metabolisms are related to a minimal metagenome, for example the biodegradation of complex sugars and glycans [2]. Profiles of human gut microbiota reflect the metabolic cooperation between different phylotypes since no genus can degrade all substrates; for example establishing all short chain fatty acids (SCFAs). SCFAs have a role in the regulation of energy metabolism, immunity and adipose tissue expansion [3]. Gut microbiota of all human beings fulfill core functions but differ in specialized functions. As a consequence some communities are linked to human diseases and obesity more than others [2].

Observations of experiments with germ-free mice compared to conventional raised mice or after conventionalization showed independent from diet a weight gain according to gut microbiota [4]. Studies on the relationship between gut microbiota composition and obesity show an increased number of Firmicutes and a reduction of Bacteroidetes in obese mice and humans compared to lean controls [4-7]. A controlled diet and weight-loss reversed the observed ratio of Firmicutes to Bacteroidetes [4,8-10]. The number of Bacteroidetes depends on the weight loss rather than on caloric intake $[7,11]$ whereas the Firmicutes group remains unchanged and no correlation with total caloric intake can be detected [10]. The ratio reaches a lean type profile after a one-year period of diet-induced weight loss [12]. The abundance of Firmicutes in diabetic persons is significantly lower compared to non-diabetics, while Bacteroidetes and Proteobacteria are

*Corresponding author: Alexander G Haslberger, Department of Nutritiona Sciences, University of Vienna, Matrasgasse 26 Vienna, Vienna 1130, Austria, Tel 00436991 2211212; E-mail: alexander.haslberger@univie.ac.at

Received January 17, 2013; Accepted February 18, 2013; Published February 22, 2013

Citation: Remely M, Dworzak S, Hippe B, Zwielehner J, Aumüller E, et al. (2013) Abundance and Diversity of Microbiota in Type 2 Diabetes and Obesity. J Diabetes Metab 4: 253. doi:10.4172/2155-6156.1000253

Copyright: @ 2013 Remely M, et al. This is an open-access article distributed under the terms of the Creative Commons Attribution License, which permits unrestricted use, distribution, and reproduction in any medium, provided the original author and source are credited. 
more abundant in diabetics. The ratio of Bacteroidetes to Firmicutes is significantly and positively correlated with reduced glucose tolerance, but negatively correlated with higher BMI. Especially, the relative abundance of Bacilli is increased in diabetic mice models and humans. Relative abundances of Actinobacteria and Verrumicrobia do not differ between diabetic and non-diabetic individuals. Bacteroides-Prevotella versus $C$. coccoides - E. rectale groups are positively associated with plasma glucose, but negatively correlated with BMI [13]. Wu et al. [12] found a higher abundance of Bacteroides (frequency of 53.6\%), and lower abundance of genus Prevotella (10.7\%), genus Proteobacteria (3.6\%) and phylum Firmicutes (10.8\%) compared to healthy volunteers in sequence analysis of selected DGGE amplicons, whereas qPCR results indicate a lower copy number of Bacteroides vulgatus and Bifidobacterium in the fecal microbiota. The Clostridium leptum cluster was more abundant in the diabetic than in the healthy group $[4,11,14]$. It is estimated that subjects with type 2 diabetes are relatively enriched with Gram-negative bacteria belonging to the phyla Bacteroidetes and Proteobacteria, which can elicit endotoxemia [13] through increased plasma LPS $[11,13,15-18]$. Obese individuals may have a microbiota more efficient in extracting energy and may be enriched in enzymes for the breakdown of otherwise indigestible alimentary polysaccharides and in other transport proteins and fermentation enzymes and further induce insulin resistance and diabetes [4]. Metabolic endotoxemia significantly correlates with oxidative stress, macrophage infiltration markers and all inflammatory markers triggering insulin resistance (PAI-1, IL-1, IL-6 and TNF- $\alpha$ ) $[4,10,15,19]$. In a high fat diet, elevated LPS increase gut permeability by affecting GLP-1, GLP-2 and PYY [10,11]. An absence of LPS receptor CD14 reduces the high-fat diet induced effects on adipose tissue inflammation, blood glucose profiles, insulin resistance index, glucose-induced insulin secretion $[7,10,15]$, hepatic steatosis, liver inflammation and adipose tissue macrophages infiltration [18]. Metabolic endotoxemia is indicated by a change in the intestinal microbiota as antibiotic treatment reduces plasma LPS levels $[7,10,11,20]$. An early modification of the microbiota with probiotics or prebiotics might be a beneficial influence [11].

The impact of the GLP-1-Agonist Liraglutide on blood glucose level, weight management and systolic blood pressure was conducted e.g. in the LEAD (Liraglutide Effect and Action in Diabetes) and in the DURATION study programs. GLP-1-Agonists lead to a glucosedependent increase of insulin secretion in beta cells and suppress the glucagon secretion in pancreatic alpha cells. Additionally, it slows the gut passage and therefore decreases the postprandial spikes in blood glucose. Furthermore, GLP-1-Agonists decrease body weight, Liraglutide showed in one study that $25 \%$ of patients lost significant weight, on average $7.7 \mathrm{~kg}$ [21].

The objective of this study was to investigate differences of gut microbiota in obese and type 2 diabetics, and the response to intervention for estimating its potential role in controlling metabolic syndrome.

\section{Materials and Methods}

\section{Ethics statement}

The project "Abundance and diversity of microbiota in type 2 diabetes and obesity" was approved by the Viennese Human Ethics committee. We received a signed form of consent from all study participants for using stool samples and data obtained from food frequency questionnaires for the analyses presented here.

\section{Study participants and study design}

Twenty-four insulin-dependent type 2 diabetes patients (aged 56 \pm 9 years)) were enrolled in cooperation with a Diabetes Oupatient Clinic. In addition we enrolled fourteen obese participants with no established insulin resistance (aged $38 \pm 14$ years) and nineteen healthy lean controls (aged $30 \pm 8$ years) (Table 1). Obese and type 2 diabetics received an intervention of nutritional counseling, type 2 diabetics an additional therapy with GLP-1-Agonists (Liraglutide). To ensure comparable data, patients were interviewed for their history of gastrointestinal diseases, use of antibiotics, probiotics or prebiotics. BMI, age and lifestyle habits were assessed in combination with a retrospective food frequency questionnaire.

Fecal samples were collected at three occasions; before treatment, during and after treatment, with a distance of 1 month between the first two time points and 3 months between the second and third time point. Controls also donated three fecal samples over the course of four months.

\section{Stool sample processing and extraction}

Stool samples were stored immediately after collection at $-18^{\circ} \mathrm{C}$ in subject's home freezer and at $-70^{\circ} \mathrm{C}$ upon arrival in the laboratory.

Total bacterial DNA was extracted from fecal samples using the QIAamp $^{\circledR}$ DNA Stool mini kit (Qiagen GmbH, Germany) according to the manufacturer's protocol. Additional, samples were treated in Fast Prep ${ }^{\mathrm{TM}}$ Lysing Matrix E tubes (MP Biodmedicals, USA) twice for $45 \mathrm{sec}$ in a bead-beater (Mini-Beadbeater 8 Bio-Spec Products, USA) with an intervening minute on ice. DNA concentration and quality was determined by picodrop (Picodrop, UK) and agarose gel-electrophoresis.

\section{Clone library}

For further analysis in $\mathrm{qPCR}$ we created clone libraries from dominant members of Akkermansia muciniphila, Prevotella intermedia and Lactobacillus casei in two stool samples of healthy volunteers. PCR-products amplified with group specific primers (Table 2) where inserted into p-GEM Easy Vector (Promega, USA) following the instructions of the manufacturer. Clone libraries were screened according to Schabereiter-Gurtner et al. [22]. The obtained nucleotide sequences from Sanger sequencing (LGC Genomics, Germany) were corrected for vector sequences and taxonomically identified using the ribosomal database project 10 (http://rdp.cme.msu.edu/).

\section{Real-time qPCR}

Bacterial abundance was quantified by qPCR using TaqMan qPCR and SYBR Green qPCR in a Rotorgene 3000 (Corbett Life Science, Australia) using 16S rDNA group specific primers (Tables 2 and 3). Specificity was checked with the Probe Match function of the ribosomal database project 10 (http://rdp.cme.msu.edu/). The PCR reaction mixture and serial DNA dilution of typically strains were prepared according to Pirkera et al. [23].

\begin{tabular}{|l|l|l|c|}
\hline Group & characterization & rate & BMI $( \pm \mathbf{S D})\left[\mathbf{k g} / \mathbf{m}^{2}\right]$ \\
\hline LC & Lean control group, no treatment & 19 & $21.78( \pm 2.35)$ \\
\hline OC & $\begin{array}{l}\text { Obese patients, intervention with } \\
\text { nutritional counseling }\end{array}$ & 14 & $33.71( \pm 3.97)$ \\
\hline D & $\begin{array}{l}\text { Type 2 diabetic, treatment with nutritional } \\
\text { counseling and GLP-1 Agonist }\end{array}$ & 24 & $38.01( \pm 5.81)$ \\
\hline
\end{tabular}

Table 1: Characterization of participant groups. 
Citation: Remely M, Dworzak S, Hippe B, Zwielehner J, Aumüller E, et al. (2013) Abundance and Diversity of Microbiota in Type 2 Diabetes and Obesity. J Diabetes Metab 4: 253. doi:10.4172/2155-6156.1000253

Page 3 of 8

\begin{tabular}{|c|c|c|c|c|c|}
\hline Target organism & Primer/Probe & Sequence (5' - 3') & Size (bp) & Conc. $[\mathrm{pmol} / \mu \mathrm{L}]$ & Reference \\
\hline \multirow{3}{*}{ All Bacteria } & Fwd primer & ACT CCT ACG GGA GGC AG & \multirow{3}{*}{468} & 10 & \multirow{3}{*}{ [1] } \\
\hline & Rev primer & GAC TAC CAG GGT ATC TAA TCC & & 10 & \\
\hline & Probe & (6-FAM)-TGC CAG CAG CCG CGG TAA TAC-(BHQ-1) & & 2 & \\
\hline \multirow{3}{*}{$\begin{array}{l}\text { Clostridium cluster IV } \\
\text { (Ruminococcaceae) }\end{array}$} & Fwd primer & GCA CAA GCA GTG GAG T & \multirow{3}{*}{239} & 4 & \multirow{2}{*}{ [2] } \\
\hline & Rev primer & CTT CCT CCG TTT TGT CAA & & 4 & \\
\hline & Probe & [3]-AGG GTT GCG CTC GTT-(BHQ-1) & & 2 & \\
\hline \multirow[t]{3}{*}{ Cluster XIVa (Lachnospiraceae) } & Fwd primer & GCA GTG GGG AAT ATT GCA & \multirow{3}{*}{477} & 5 & [4] \\
\hline & Rev primer & CTT TGA GTT TCA TTC TTG CGAA & & 5 & \\
\hline & Probe & (6-FAM)-AAA TGA CGG TAC CTG ACT AA-(BHQ-1) & & 1,5 & \\
\hline \multirow{3}{*}{ Bacteroidetes } & Fwd primer & GAG AGG AAG GTC CCC CAC & \multirow{3}{*}{106} & 3 & \multirow{3}{*}{ [5] } \\
\hline & Rev primer & CGC TAC TTG GCT GGT TCA G & & 3 & \\
\hline & Probe & $\begin{array}{l}\text { (6-FAM)-CCA TTG ACC AAT ATT CCT CAC TGC TGC } \\
\text { CT-(BHQ-1) }\end{array}$ & & 1 & \\
\hline \multirow[b]{3}{*}{ Bifidobacterium spp. } & Fwd primer & GCG TGC TTAACA CAT GCA AGT C & \multirow[b]{3}{*}{125} & 3 & \multirow[b]{3}{*}{ [6] } \\
\hline & Rev primer & CAC CCG TTT CCA GGA GCT ATT & & 3 & \\
\hline & Probe & $\begin{array}{l}\text { (6-FAM)-TCA CGC ATT ACT CAC CCG TTC GCC- } \\
(B H Q-1)\end{array}$ & & 1.5 & \\
\hline \multirow[t]{3}{*}{ Archaea } & Fwd primer & ATT AGA TAC CCG GGT AGT CC & & 4 & [7] \\
\hline & Rev primer & GCC ATG CAC CWC CTC T & $1044-1059$ & 4 & \multirow{2}{*}{ [1] } \\
\hline & Probe & (6-FAM)-AGG AAT TGG CGG GGG AGC AC(BHQ-1) & $915-934$ & 4 & \\
\hline
\end{tabular}

Table 2: Primers and TaqMan ${ }^{\circledR}$-probes targeting $16 \mathrm{~S}$ rDNA coding regions of bacteria and archaea.

\begin{tabular}{|c|c|c|c|c|c|}
\hline Target organism & Primer & Sequence $\left(5^{\prime}-3^{\prime}\right)$ & Size (bp) & Conc. [pmol/ $/ \mu \mathrm{L}]$ & Reference \\
\hline \multirow{2}{*}{ Lactic Acid Bacteria (LAB) } & Fwd primer & AGC AGT SGG GAA TCT TCC A & \multirow{2}{*}{$352-700$} & 4 & \multirow{2}{*}{ [8] } \\
\hline & Rev primer & ATT YCA CCG CTA CAC ATG & & 4 & \\
\hline \multirow{2}{*}{ BcoAT gene } & Fwd primer & GCI GAI CAT TTC ACI TGG AAY WSI TGG CAY ATG & \multirow{2}{*}{$\sim 540$} & 27 & \multirow{2}{*}[9]{} \\
\hline & Rev primer & CCT GCC TTT GCA ATR TCI ACR AAN GC & & 27 & \\
\hline \multirow{2}{*}{ Enterobacteria } & Fwd primer & AGC ACC GGC TAA CTC CGT & $492-509$ & 3 & [10] \\
\hline & Rev primer & GAA GCC ACG CCT CAA GGG CAC AA & $834-856$ & 3 & [11] \\
\hline \multirow[t]{2}{*}{ Prevotella } & Fwd primer & CACCAAGGCGACGATCA & \multirow{2}{*}{1458} & 2,5 & \multirow{2}{*}[12]{} \\
\hline & Rev primer & GGATAACGCCYGGACCT & & 2,5 & \\
\hline \multirow{2}{*}{ Akkermansia } & Fwd primer & CAGCACGTGAAGGTGGGGAC & \multirow{2}{*}{1505} & 2,5 & \multirow{2}{*}{ [13] } \\
\hline & Rev primer & CCTTGCGGTTGGCTTCAGAT & & 2,5 & \\
\hline
\end{tabular}

Table 3: Primers (SYBR ${ }^{\circledR}$ Green) targeting $16 \mathrm{~S}$ rDNA coding regions of bacteria and butyryl-coenzyme $A(C o A)$ CoA transferase genes.

\section{PCR/DGGE}

The diversity of total bacteria was measured by DGGE using the primer set 341f-GC 5'-CCT ACG GGA GGC AGC AG-3' [24] and 518r 5'-ATT ACC GCG GCT GCT GG-3' [25]. The endpoint PCR was carried out with a ready-to-use GoTaq ${ }^{\circledR}$ Green Master Mix (Promega, USA) with $1.5 \mathrm{mM} \mathrm{MgCl}_{2}$ in a 96-well Gradient Thermal Cycler MultiGene ${ }^{\mathrm{TM}}$, Labnet International, Inc., USA).

DGGE gels were prepared as described previously [26] with a linear gradient of $25-65 \%$ for total bacteria using a peristaltic pump. The reference marker contained 16S rDNA gene fragments of Lactobacillus reuteri DSM 20016, Escherichia coli 1029, Enterococcus faecium DSM 20477, Clostridium blautiacoccoides DSM 935, Clostridium leptum DSM 753, Bifdobacteriumlongum sp. longum DSM 20219 and Bacteriodes theataiomicron DSM 2079.

\section{High throughput sequencing}

Sixteen samples were analyzed with Roche GS FLX+ Titanium next generation sequencing ((LGC sequencing GmbH, Germany). For alignment and further analyses we used the pyro pipeline of the ribosomal database project 10 (http://rdp.cme.mus.edu/) and qiime. We then performed significance tests, UPGMA clustering, and principal coordinate analysis in qiime. We used jackknifing to determine results.

\section{Statistical analysis}

We used the non-parametric Mann-Whitney $U$ test and for three unpaired groups the non-parametric Kruskal Wallis ANOVA for comparison of the non-parametric unpaired values in OriginPro version 8 OriginLab, USA. P values $<0.05$ were determined as statistically significant. DGGE-gels were evaluated with Gel compare II (Applied Maths, Belgium).

\section{Results}

All used methods indicate high individual variability. However the variation within samples of the same individual over time was consistently lower than variation between subjects. Our approach was to first compare the abundance of each bacterial group individually, then to calculate the Firmicutes to Bacteroidetes ratio and finally to compare our results with discussed enterotypes. We applied PCRDGGE fingerprinting to estimate the species richness of all bacteria and selected sixteen samples for 454 sequencing to obtain in-depth data on microbial diversity.

\section{Analyses of quantitative GI microbiota composition}

We did not detect any differences between the three groups in total bacterial abundance. However, the microbial composition 
showed significant differences between type 2 diabetics, lean controls and obese participants. The most abundant bacterial groups in lean, obese and type 2 diabetics were the Gram-positive bacteria belonging to the Clostridium Cluster IV and the Clostridium Cluster XIVa as well as the Gram-negative Bacteroidetes, which altogether made up a median proportion of more than $95 \%$ of all detected bacteria. The ratio of Firmicutes:Bacteroidetes increased in type 2 diabetics under dietary intervention and therapy with GLP-1-Agonist during the study period (T3 p=0.04) from an already higher Firmicutes:Bacteroidetes ratio at the first time point $(\mathrm{p}=0.02)$. In the obese and lean groups, a lower Firmicutes:Bacteroidetes ratio correlated with lower BMI. Clostridium Cluster IV and Clostridium Cluster XIVa abundances were not significantly different between the groups or time points, but we observed a decrease in intervention of type 2 diabetics. Lactobacilli were significantly more abundant in type 2 diabetics at baseline $(\mathrm{p}=0.01)$ and increased over the study period (T3 $\mathrm{p}=0.004$; Figure 1$)$, compared to the other groups. High throughput sequencing confirmed these findings. Overall type 2 diabetics were enriched in Firmicutes especially Clostridiales and Bacilli, especially Lactobacillales. In accordance Bacteroidetes were less abundant in diabetics, resulting in a higher Firmicutes:Bacteroidetes ratio. Between the first and third time point Bacteroidetes decreased and Firmicutes increased. Sequencing and $\mathrm{qPCR}$ were congruent in finding a Firmicutes:Bacteroidetes ratio of 1 in lean controls (Figures 2 and 3). No significant changes could be detected within the butyryl-CoA: acetate CoA-transferase gene abundance between the study groups, this butyrate production gene tended to be more abundant in type 2 diabetics.

Archaea as well as species of the genera bifidobacteria, enterobacteria, Akkermansia and Prevotella accounted for the remaining proportion of the microbiota. Enterobacteria were more abundant in type 2 diabetics at all time points compared to the control group. This observation was significant at the first two time points despite greater variability in diabetics $(\mathrm{T} 1 \mathrm{p}=0.04 ; \mathrm{T} 2 \mathrm{p}=0.01)$. Greater abundance of enterobacteria was also detected in obese study participants. We observed no significant differences of Prevotella and bifidobacteria between the three groups or time points. Akkermansia

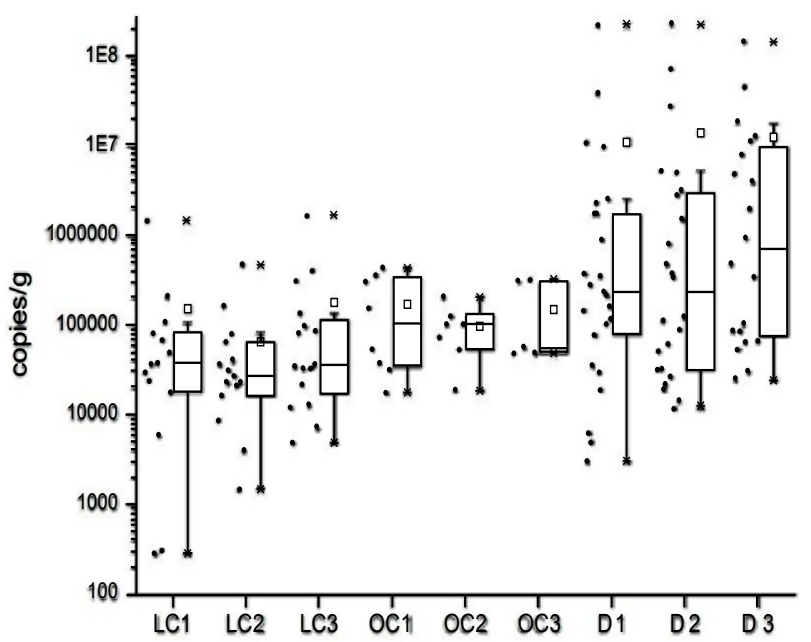

Figure 1: 16S rDNA qPCR quantification with primer pair for lactic acid bacteria showing significant higher abundance in type 2 diabetics (D) than obese patients (OC) and lean controls (LC).

( $\mathrm{T} 1$, samples taken before intervention; T2, samples taken after 1 month of intervention; $\mathrm{T} 3$, samples taken after 4 month of intervention)

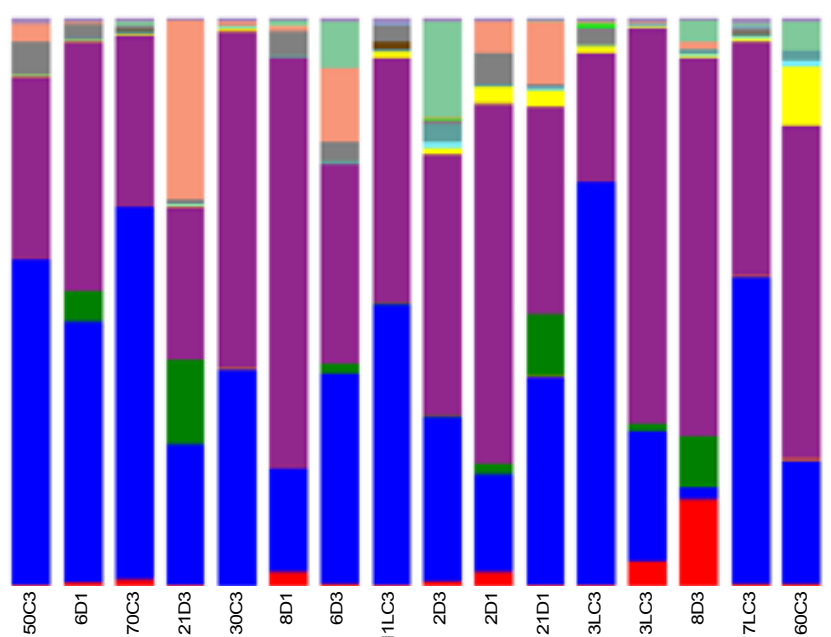

Bacteria;Actinobacteria;Actinobacteria

Bacteria;Bacteroidetes;Bacteroidia

Bacteria;Bacteroidetes;Other

Bacteria;Firmicutes;Bacilli

Bacteria;Firmicutes;Clostridia

Bacteria;Firmicutes;Erysipelotrichi

Bacteria;Firmicutes;Other

Bacteria;Fusobacteria;Fusobacteria

Bacteria;Other;Other

Bacteria;Proteobacteria;Alphaproteobacteria

Bacteria;Proteobacteria;Betaproteobacteria

Bacteria;Proteobacteria;Deltaproteobacteria

Bacteria;Proteobacteria;Gammaproteobacteria

Bacteria;Proteobacteria;Other

Bacteria;Synergistetes;Synergistia

Bacteria;Verrucomicrobia;Verrucomicrobiae

Unassignable;Other;Other

Unclassified;Other;Other

Figure 2: Bar Charts of 454 sequencing results reflect qPCR analysis with increasing abundance of Firmicutes especially Clostridiales and Bacilli in type 2 diabetics (D) resulting in an increasing ratio of Firmicutes:Bacteroidetes between the time points ( $T 1$, samples taken before intervention; T3, samples taken after 4 month of intervention). Verrumicrobia and enterobacteria are also higher abundant in type 2 diabetics and enriched in obese patients (OC) Lean controls (LC) showed an according lower abundance of named bacterial groups and higher abundance of Bacteroidetes.

increased throughout the study period in type 2 diabetics with an already higher initial abundance compared to lean controls and obese subjects in data obtained from qPCR as well as in 454 sequencing results $(\mathrm{p}=0.16)$.

At all three time points, Archea were significantly ( $\mathrm{p}=0.05)$ more frequent in the type 2 diabetics ( $18 \pm 5$ patients) compared to control groups, followed by obese patients with ( $4 \pm 0$ participants) and lean controls ( $5 \pm 3$ participants).

\section{Diversity analyses}

DGGE fingerprinting indicated highly diverse individual differences of the microbiota. The dataset was subjected to principal component analysis (PCA). The first two principal components (PCs) explained $15.6 \%$ and $12.0 \%$ of variance (data not shown). Although overlapping, PCA showed grouping of band patterns according to the three groups of study participants. Between the groups a ranking of diversity was observed, as type 2 diabetes patients had the lowest diversity, followed by obese participants and the highest diversity in 


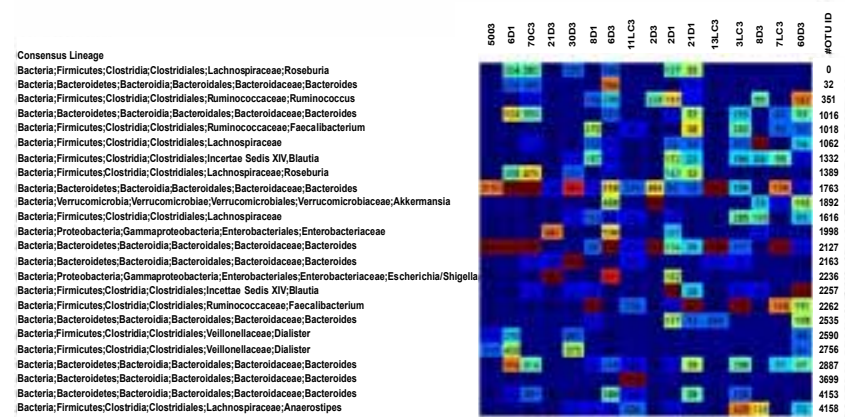

Figure 3: Heatmap showing abundances within the 454 sequencing dataset between the groups and time points $(\mathrm{OC}$ - obese patients, $\mathrm{LC}$ - lean control; D1 - type 2 diabetics time point one; D3-type 2 diabetics time point 3) The OTU heatmap displays raw OTU counts per sample, where the counts are colored based on the contribution of each OTU to the total OTU count present in that sample, blue indicates contributions with low percentage of OTUs to sample, whereas red contributes for a high percentage of OTUs.

the lean control group. Differences between type 2 diabetics and lean controls were significant at time point $2(\mathrm{p}=0.03)$ and time point 3 $(\mathrm{p}=0.01)$ (Table 4).

Shannon's diversity index of 454 sequencing results showed no significant differences between the three groups (D 5.19 \pm 2.62 ; LC 6.48 \pm 0.47 ; OC $4.51 \pm 2.60)$. Lean controls had a higher Shannon index and a higher number of sequences $(683.3 \pm 59.6)$ compared to obese controls (571.5 \pm 48.2$)$ and type 2 diabetics (T1 $645.5 \pm 22.6$; T3 619.0 $\pm 62.3)$.

PCA was also calculated on the 454 sequencing data. The first three PCs explained $44.6 \%, 17.2 \%$ and $8.3 \%$ of the variance in the data. Samples of the diabetic group, obese group and the control group at phylum level were clustered along PC 1 and PC 2 (Figure 4). In the PCA of sequencing results, samples of one participant at the two different time points and also the three different groups were grouped according to their variance along principal components. The first PCA was attributed to Firmicutes and Verrumicrobia, it contributed most to the separation along PC1 and Proteobacteria, Actinobacteria and Bacteroides contributed to the clustering along PC2. PCA of multiple genera showed grouping of the subjects with diabetes (Figure 4). Additionally Jackknifing did not affect the UPGMA unifrac clustering results (Figure 5).

\section{Food Frequency Questionnaire (FFQ)}

Analysis of the participant's FFQ did not reveal any differences in the consumption of grain, vegetables, fruits, meat, dietary products and fish. Mostly type 2 diabetics consumed sweets less than once a week $(40.0 \% \mathrm{~T} 1,28.0 \% \mathrm{~T} 2)$ in contrast $28.0 \%$ of lean individuals consumed sweets three to five times per week and $50.0 \%$ of obese controls consumed sweets one to three times per week. However, study participants stated an average consumption of sweets 1-15 times per week. The main differences are seen in life style patterns due to the lower physical activity in obese $(37.5 \% \mathrm{~T} 1,12.5 \% \mathrm{~T} 3$ never do sports) and type 2 diabetics (64.0\% T1, 52.0\% T3 never do sports), whereas $44.0 \%$ of lean controls did sports 1-3 times per week. $\mathrm{HbA}_{1 \mathrm{C}}$ blood level was determined at laboratory Health Center South in Vienna. The levels were between $7-8 \%$ in our type 2 diabetes group. 19 out of 24 type 2 diabetes patients showed an average of $3.5 \%( \pm 1.9)$ of weight reduction.

\section{Discussion}

This study focused on the characteristics of fecal microbiota in type 2 diabetic patients before and after intervention with nutritional counseling and therapy with GLP-1-Agonist compared to a group of obese volunteers receiving nutritional counseling and a lean group.

\section{Similar counts of total bacterial abundance}

In this study we did not observe any significant differences in total bacterial abundance between type 2 diabetes, obese and lean controls as a possible result of the intervention. Accordingly, Larsen et al. detected similar counts of total bacterial abundance in type 2 diabetics and the control group [13]. Bifidobacteria showed no significant differences between the three groups and intervention, possibly reflecting no significant differences in food composition or as an indicator for the well-adjusted $\mathrm{HbA}_{1 \mathrm{c}}$ values. Wu et al. reports a reduced abundance of bifidobacteria in type 2 diabetics in qPCR as well as in sequencing results as a possible characteristic of diabetes [12]. In mice, a significant negative correlation has been observed between endotoxemia and Bifidobacterium spp., but no relationship with any other bacterial

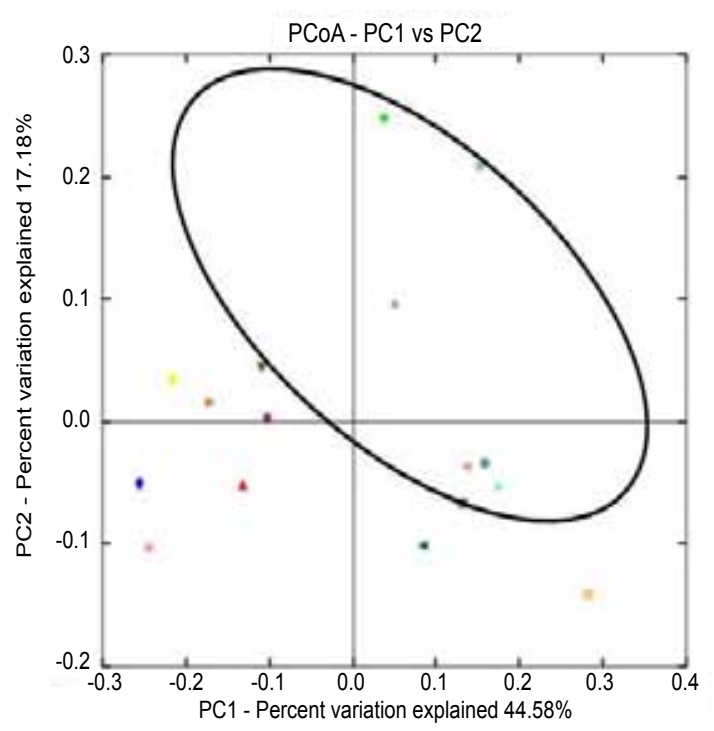

Figure 4: PCA based on dominant bacteria of PC1 (Firmicutes and Verrumicrobia) and of PC2 (Proteobacteria, Actinobacteria and Bacteroides) illustrating grouping of type 2 diabetic samples (ellipse), as a result of a higher ratio of Firmicutes:Bacteroidetes in type 2 diabetics, lower abundance of Verrumicrobia and higher abundance of Proteobacteria.

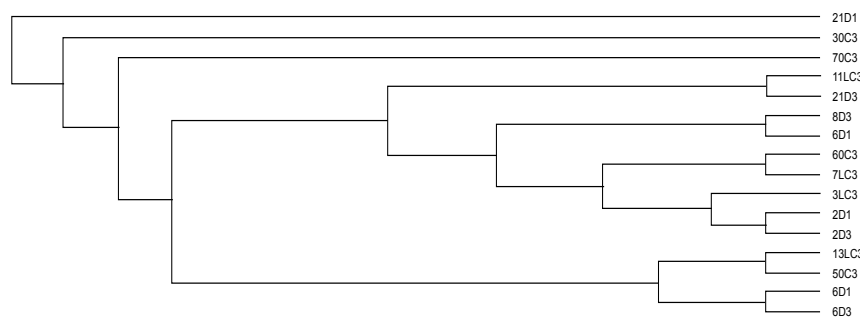

Figure 5: Jackknifing did not affect UPGMA clustering results as groups and patients are less different compared to differences between the three groups (LC, lean control group; OC, obese patients). Type 2 diabetics (D) are grouped between the two time points ( $\mathrm{T} 1$, samples taken before intervention; T3, samples taken after 4 month of intervention). 
group during treatment with high fat diet and oligofructose [27]. A treatment with prebiotics may improve or prevent disruption of intestinal epithelial barrier function, reduce intestinal permeability and consequently improve endotoxemia [20].

\section{Moderate increase of diversity in type 2 diabetics with intervention}

More than the abundance of total bacteria or certain bacterial groups, the diversity of microbiota indicated by richness of species or gene counts is now seen as an important indicator of microbiota resilience and gut health [28]. Shannon's diversity index showed no significant differences between the groups, whereas band pattern analysis showed significant differences between the lean control group and type 2 diabetics. In type 2 diabetics an increase of bands was seen with intervention whereas the values of lean controls remained unaffected (Table 4). Wu et al. does not observe significant differences by comparing diversity profiles of type 2 diabetics and non-diabetics, which indicates an association of health and disease with a shift in the balance of gut microbiota rather than an action of a single microbe affecting the diversity [12]. Ley et al. observes a lower diversity in ob/ob animals with lower abundance of Bacteroidetes and a proportional increase in Firmicutes compared to lean mice. In this model, the diversity is affected by obesity [29], however we indicated a lower diversity in type 2 diabetics, but not in obese volunteers. In agreement to our observed PCs, PC1 Firmicutes and Verrumicrobia, PC2 Proteobacteria, Actinobacteria and Bacteroidetes (Figure 4), Larsen et al. shows also a higher separation between the groups attributed to Bacteroides, Firmicutes and Verrumicrobia (45\%) and in second direction the combination of Proteobacteria and Actinobacteria (28\%) [13]. More than changes in the abundance of some microbiota groups or the small changes in body weight in some patients this increase In the diversity could indicate the success of the nutritional/ GLP-1-Agonist intervention.

\section{Higher ratio of Firmicutes versus Bacteroidetes in type 2 diabetes patients}

We detected a higher abundance of Firmicutes in type 2 diabetics. Firmicutes $(60 \%$ at T3) increased and Bacteroidetes (35\% at T3) decreased during intervention. The phylum Fimicutes harbours many butyrate and acetat producers. According to previous studies with higher concentration of fermentation end products in tissue and lower energy content in feces, butyryl-CoA: acetate CoA-transferase genes tended to be more abundant in type 2 diabetics, but this difference was not significant. Obese patients on the contrary showed a decreasing ratio of Firmicutes (55\%) to Bacteroidetes (45\%; Figures 2,3). Intervention here resulted in a weight loss of $(4.87 \pm 3.68 \mathrm{~kg})$. Weight loss was highly diverse between individuals.

Consequences of these shifts remain under discussion for their effects on endotoxin mediated low grade inflammation, but also for consequences on energy extraction and body weight. An increased Firmicutes to Bacteroidetes ratio is previously associated with increased body weight [6] but also controversial data exist, e.g. by higher

\begin{tabular}{|l|c|c|c|}
\hline time point & LC & OC & D \\
\hline T1 & $12.1 \pm 3.5$ & $10.5 \pm 3.6$ & $8.5 \pm 4.4$ \\
\hline T2 & $13.3 \pm 4.9$ & $9.0 \pm 1.6$ & $9.4 \pm 3.4$ \\
\hline T3 & $14.0 \pm 4.0$ & $10.3 \pm 5.6$ & $9.6 \pm 3.6$ \\
\hline
\end{tabular}

LC: Lean Controls; OC: Obese Participants; D: Type 2 Diabetics; T: Time Point

Table 4: Number of bands observed in PCR-DGGE fingerprinting in lean controls, obese patients and type 2 diabetics at all three time points.
Clostridia in association with higher plasma glucose [13]. A higher ratio of Firmicutes versus Bacteroidetes increases the energy harvest and the content of SCFA and as such the lipogenesis and accumulation in adipocytes [11]. Schwiertz et al. on the contrary reports a lower Firmicutes to Bacteroidetes ratio in overweight human adults compared to lean controls [8] whereas other working groups do not find an association between the Bacteroidetes to Firmicutes ratio and BMI $[9,20,30,31]$.

Not only obesity linked microbiota characteristics, but also changes in microbiota composition during weight loss are under discussion. It has been suggested that the abundance of Bacteroidetes responds to the weight loss whereas the Firmicutes remain unaffected [32]. Recently published data show that especially Firmicutes promote caloric extraction from the diet in zebra-fish. Eating encourages the growth of Firmicutes and the fat storage in intestinal cells. Firmicutes decrease during hypocaloric diets and Bacteroidetes get the chance to proliferate [33]. Larsen et al. shows a positive correlation between plasma glucose levels and Bacteroidetes to Firmicutes ratio [13]. As we approved the $\mathrm{HbA}_{1 \mathrm{c}}$ blood levels of our type 2 diabetic patients, we had no possibility to approve this correlation. After the start of intervention with GLP-1-Agonist all type 2 diabetics had well-adjusted $\mathrm{HbA}_{1 \mathrm{C}}$ blood levels of 7-8\%, a value that according to American Diabetes Association protects against long-term consequences [34].

\section{Higher lactic acid bacteria (LAB) may contribute to chronic inflammation?}

Our type 2 diabetes patients harbored higher proportions of LAB according to $\mathrm{qPCR}$ analysis. This was confirmed by sequencing for higher abundance of Enterococcus, Streptococcus and Lactobacillus. Furthermore, LAB increased in type 2 diabetics during the study period (Figure 1).

LAB are very well known but also subject of controversy [3]. They represent a very heterogeneous group with well documented immune modulating qualities, which may potentially contribute to chronic inflammation in type 2 diabetes [13]. The relative abundance of Bacilli is increased in diabetics; especially the Lactobacillus group is enriched in type 2 diabetic mice models. Many bacterial isolates received from diabetic patients are able to produce toxines, indicating the necessity for virulence of these bacteria in blood stream infections [35]. On the other hand several strains of Lactobacillus are tested as probiotics, e.g. L. paracasei ssp. Paracasei F19 and L. gasseri SBT2055to decrease fat mass (visceral and subcutaneous).

Therefore, increases in $\mathrm{LAB}$ can be interpreted as immune modulating, even inflammatory process or an immune mechanisms underlying body-weight management.

\section{Higher enterobacteria in type 2 diabetics - contributors to bacteremia?}

Type 2 diabetics and to a lesser extent also obese volunteers showed a higher abundance of enterobacteria, especially E. coli. Enterobacteria are of special interest in type 2 diabetes as enterobacteria are a major cause of morbidity and mortality in type 2 diabetics [36]. Thomsen et al. observed a 3-fold increased risk to E. coli and other enterobacteria. Diabetic patients aged $<45$ years have a 15 -fold higher risk for acute pyelonephritis and diabetics aged $>65$ had 3-6 times higher risk compared to non-diabetics. A diabetes prevalence of $20-30 \%$ was previously reported among patients with enterobacterial bacteremia [37]. 
Citation: Remely M, Dworzak S, Hippe B, Zwielehner J, Aumüller E, et al. (2013) Abundance and Diversity of Microbiota in Type 2 Diabetes and Obesity. J Diabetes Metab 4: 253. doi:10.4172/2155-6156.1000253

\section{Caloric restriction increases abundance of Akkermansia and Archaea?}

We report an enhanced abundance of Akkermansia (Figures 2 and 3 ) and a higher appearance of Archea in the type 2 diabetes group. Only few lean controls harbored Archaea.

On the basis of Mucin-degradation, Akkermansia do not compete with the other microbiota and are not dependent on nutrients deriving from host food consumption [38]. The observed higher abundance of Akkermansia in type 2 diabetics in our study may reflect a better adaption to caloric restriction compared to the rest of intestinal microbiota as they do not depend on host diet. An increased abundance of A. muciniphila has also been observed in hamsters lacking foodderived enteral nutrients [39].

Archaea have been reported to be slightly more abundant in obesity and anorexic patients and were discussed to adapt towards optimal exploitation of hypocaloric diet [31]. Therefore, Archaea are indirect promoters of caloric intake by the colon and further fat accumulation [40]. Studies in humans show that removal of $\mathrm{H}_{2}$ by the synothropic relationship with bacteria improves fermentation efficiency $[7,41]$. Moreover less energy has been measured in obese feces in mice relative to lean littermates [41]. The symbiotic relationship might maximize the microbiota's ability to generate energy from otherwise non-digestible food components, thereby they get enriched during caloric restriction to guarantee an adequate caloric intake of the host and as such complicate or even prevent weight reduction over time.

\section{Prevotella reflect same compositional diet between the three groups}

Our study participants showed no differences in Prevotella abundance over time, as our type 2 diabetics were well adjusted to $\mathrm{HbA}_{1}$ blood levels. In contrast Larsen et al. reports significant higher ratios of Bacteroides-Prevotella group versus class Clostrida and C. coccoides- E. rectale group in type 2 diabetics. Clostridium ssp., C. coccoides decrease and Bacteroides-Prevotella group increase with body weight loss [13].

Prevotella are also of special interest because of their role in the formation of discussed gut enterotypes. First of all we made the distribution into the nowadays confirmed two enterotypes (Bacteroides-Akkermansia or Prevotella) with no observable difference. However, if we have a detailed view with the distribution of Bacteroides and Akkermansia in each enterotype, type 2 diabetesis is more related to enterotype 3 and obese particpants and the lean control group to enterotype 1, which might reflect the dependence of an enterotype on caloric restriction although both are related to a Western diet.

On the other hand, Oluf Pedersen indicates that obese and "metabolically unhealthy" people tend to have the Bacteroides enterotype, so the classification may get very important to ensure an individual therapy and treatment [42].

\section{Conclusion}

Gut microbiota composition and activity is a key element in controlling vital functions of the host and also the nutritional status. Type 2 diabetes and obesity are associated with changes in its abundance and diversity. Microbiota interacts with functions underlying inflammation, energy extraction as well as the gut barrier. Our results indicate the necessity to concentrate on gut microbiota host interaction to better understand the involvement of gut microbiota in the development of obesity and type 2 diabetes.

\section{Acknowledgment}

The work was supported by the Austrian Research Promotion Agency (FFG) and the Austrian Science Fund (FWF; Projectnumber: L 574-B13). The authors thank all the staff of the diabetes clinic at the Health Centre South and the head of the laboratory at the health center, Dr. Ernst Forster. Special thanks to all the participating subjects without whom this study would not have been possible.

\section{References}

1. Verdu EF (2009) Probiotics effects on gastrointestinal function: beyond the gut? Neurogastroenterol Motil 21: 477-480.

2. Gerritsen JJ, Smidt HH, Rijkers GT, de Vos WM (2011) Intestinal microbiota in human health and disease: the impact of probiotics. Genes Nutr 6: 209-240.

3. Delzenne NM, Cani PD (2011) Interaction between obesity and the gut microbiota: relevance in nutrition. Annu Rev Nutr 31: 15-31.

4. Musso G, Gambino R, Cassader M (2010) Obesity, diabetes, and gut microbiota: the hygiene hypothesis expanded? Diabetes Care 33: 2277-2284.

5. Ley RE, Turnbaugh PJ, Klein S, Gordon JI (2006) Microbial ecology: human gut microbes associated with obesity. Nature 444: 1022-1023.

6. Turnbaugh PJ, Ley RE, Mahowald MA, Magrini V, Mardis ER, et al. (2006) An obesity-associated gut microbiome with increased capacity for energy harvest. Nature 444: 1027-1031.

7. DiBaise JK, Zhang $\mathrm{H}$, Crowell MD, Krajmalnik-Brown $\mathrm{R}$, Decker GA, et al. (2008) Gut microbiota and its possible relationship with obesity. Mayo Clin Proc 83: $460-469$.

8. Schwiertz A, Taras D, Schäfer K, Beijer S, Bos NA, et al. (2010) Microbiota and SCFA in lean and overweight healthy subjects. Obesity (Silver Spring) 18 : 190-195.

9. Duncan SH, Lobley GE, Holtrop G, Ince J, Johnstone AM, et al. (2008) Human colonic microbiota associated with diet, obesity and weight loss. Int $\mathrm{J}$ Obes (Lond) 32: 1720-1724.

10. Cani PD, Bibiloni R, Knauf C, Waget A, Neyrinck AM, et al. (2008) Changes in gut microbiota control metabolic endotoxemia-induced inflammation in high-fat diet-induced obesity and diabetes in mice. Diabetes 57: 1470-1481.

11. Diamant M, Blaak EE, de Vos WM (2011) Do nutrient-gut-microbiota interactions play a role in human obesity, insulin resistance and type 2 diabetes? Obes Rev 12: $272-281$

12. Wu X, Ma C, Han L, Nawaz M, Gao F, et al. (2010) Molecular characterisation of the faecal microbiota in patients with type II diabetes. Curr Microbiol 61 $69-78$

13. Larsen N, Vogensen FK, van den Berg FW, Nielsen DS, Andreasen AS, et al (2010) Gut microbiota in human adults with type 2 diabetes differs from nondiabetic adults. PLoS One 5: e9085.

14. Bäckhed F, Ding H, Wang T, Hooper LV, Koh GY, et al. (2004) The gut microbiota as an environmental factor that regulates fat storage. Proc Nat Acad Sci U S A 101: 15718-15723.

15. Cani PD, Delzenne NM (2009) The role of the gut microbiota in energy metabolism and metabolic disease. Curr Pharm Des 15: 1546-1558.

16. Luoto R, Kalliomäki M, Laitinen K, Isolauri E (2010) The impact of perinata probiotic intervention on the development of overweight and obesity: follow-up study from birth to 10 years. Int J Obes (Lond) 34: 1531-1537.

17. Tsukumo DM, Carvalho BM, Carvalho-Filho MA, Saad MJ (2009) Translational research into gut microbiota: new horizons in obesity treatment. Arq Bras Endocrinol Metabol 53: 139-144.

18. Cani PD, Delzenne NM, Amar J, Burcelin R (2008) Role of gut microflora in the development of obesity and insulin resistance following high-fat diet feeding Pathol Biol (Paris) 56: 305-309.

19. Caesar R, Fåk F, Bäckhed F (2010) Effects of gut microbiota on obesity and atherosclerosis via modulation of inflammation and lipid metabolism. J Intern Med 268: 320-328.

20. Cani PD, Delzenne NM (2009) Interplay between obesity and associated metabolic disorders: new insights into the gut microbiota. Curr Opin Pharmaco 9: 737-743.

21. Nauck M, Frid A, Hermansen K, Shah NS, Tankova T, et al. (2009) Efficacy and 
Citation: Remely M, Dworzak S, Hippe B, Zwielehner J, Aumüller E, et al. (2013) Abundance and Diversity of Microbiota in Type 2 Diabetes and Obesity. J Diabetes Metab 4: 253. doi:10.4172/2155-6156.1000253

Page 8 of 8

safety comparison of liraglutide, glimepiride, and placebo, all in combination with metformin, in type 2 diabetes: the LEAD (liraglutide effect and action in diabetes)-2 study. Diabetes Care 32: 84-90.

22. Schabereiter-Gurtner C, Piñar G, Lubitz W, Rölleke S (2001) An advanced molecular strategy to identify bacterial communities on art objects. J Microbiol Methods 45: 77-87.

23. Pirkera A, Stockenhuberb A, Remelya M, Harranta, Hippea B, et al. (2012) Effects of antibiotic therapy on the gastrointestinal microbiota and the influence of Lactobacillus casei. Food Agric Immunol.

24. Muyzer G, de Waal EC, Uitterlinden AG (1993) Profiling of complex microbial populations by denaturing gradient gel electrophoresis analysis of polymerase chain reaction-amplified genes coding for $16 \mathrm{~S}$ rRNA. Appl Environ Microbiol 59: 695-700.

25. Neefs JM, Van de Peer Y, De Rijk P, Goris A, De Wachter R (1991) Compilation of small ribosomal subunit RNA sequences. Nucleic Acids Res 19: 1987-2015.

26. Muyzer G, Smalla K (1998) Application of denaturing gradient ge electrophoresis (DGGE) and temperature gradient gel electrophoresis (TGGE) in microbial ecology. Antonie Van Leeuwenhoek 73: 127-141.

27. Cani PD, Neyrinck AM, Fava F, Knauf C, Burcelin RG, et al. (2007) Selective increases of bifidobacteria in gut microflora improve high-fat-diet-induced diabetes in mice through a mechanism associated with endotoxaemia. Diabetologia 50: 2374-2383.

28. Turnbaugh PJ, Hamady M, Yatsunenko T, Cantarel BL, Duncan A, et al. (2009) A core gut microbiome in obese and lean twins. Nature 457: 480-484.

29. Ley RE, Bäckhed F, Turnbaugh P, Lozupone CA, Knight RD, et al. (2005) Obesity alters gut microbial ecology. Proc Natl Acad Sci U S A 102: 1107011075

30. Abdolrasulnia M, Menachemi N, Shewchuk RM, Ginter PM, Duncan WJ, et al. (2008) Market effects on electronic health record adoption by physicians. Health Care Manage Rev 33: 243-252.

31. Arumugam M, Raes J, Pelletier E, Le Paslier D, Yamada T, et al. (2011) Enterotypes of the human gut microbiome. Nature 473: 174-180.
32. Turnbaugh PJ, Gordon JI (2009) The core gut microbiome, energy balance and obesity. J Physiol 587: 4153-4158.

33. Semova I, Carten JD, Stombaugh J, Mackey LC, Knight R, et al. (2012) Microbiota regulate intestinal absorption and metabolism of fatty acids in the zebrafish. Cell Host Microbe 12: 277-288.

34. Association AD (2010) Executive Summary: Standards of Medical Care in Diabetes-2010. Diabetes Care 33: S4-S10.

35. Hancock IE (2000) Pathogencity of enterococci, in ASM publications: 751-758

36. Graff LR, Franklin KK, Witt L, Cohen N, Jacobs RA, et al. (2002) Antimicrobial therapy of gram-negative bacteremia at two university-affiliated medical centers. Am J Med 112: 204-211.

37. Thomsen RW, Hundborg HH, Lervang HH, Johnsen SP, Schønheyder HC et al. (2005) Diabetes mellitus as a risk and prognostic factor for communityacquired bacteremia due to enterobacteria: a 10-year, population-based study among adults. Clin Infect Dis 40: 628-631.

38. Derrien M, Van Baarlen P, Hooiveld G, Norin E, Müller M, et al. (2011) Modulation of Mucosal Immune Response, Tolerance, and Proliferation in Mice Colonized by the Mucin-Degrader Akkermansia muciniphila. Front Microbiol 2 166.

39. Sonoyama K, Fujiwara R, Takemura N, Ogasawara T, Watanabe J, et al. (2009) Response of gut microbiota to fasting and hibernation in Syrian hamsters. App Environ Microbiol 75: 6451-6456.

40. Dridi B, Raoult D, Drancourt M (2011) Archaea as emerging organisms in complex human microbiomes. Anaerobe 17: 56-63.

41. Gill SR, Pop M, Deboy RT, Eckburg PB, Turnbaugh PJ, et al. (2006) Metagenomic analysis of the human distal gut microbiome. Science 312: 13551359 .

42. Yong Ed (2012) Gut microbial 'enterotypes' become less clear-cut. Nature. 